A C T A C H E M I C A S C A N D I N A V.I C A $22 \quad$ (1968) $444-452$

\title{
Least Squares Analysis of NMR-Spectra of Pair-Powders
}

\author{
B J ØR N P E D ERSEN
}

Sentralinstitutt for industriell forskning, Oslo 3, Norway

\begin{abstract}
The structural information obtainable from fitting a theoretical spectrum by least squares methods to a proton magnetic resonance spectrum of a powder where the resonant nuclei are situated in equivalent pairs (pair-powders) is explored. The theoretical spectrum (Pake, ${ }^{4}$ ) depends on only two factors of physical significance, $\alpha$ and $\beta$, where $\alpha$ depends only on the intrapair coupling and $\beta$ only on the interpair coupling. The successful fitting of a theoretical spectrum to an experimental spectrum leads, therefore, to an unambiguous division of the Van Vleck second moment in an intrapair part, 4/5 $\alpha^{2}$, and an interpair part, $\boldsymbol{\beta}^{2}$. The use of a finite modulation amplitude, $H_{\mathrm{M}}$, is shown experimentally only to affect $\beta^{2}$ which increases linearly as $\left(\frac{1}{2} H_{\mathrm{M}}\right)^{2}$.

The theoretical spectra are in good agreement with the experimental spectra of $\mathrm{CaSO}_{4} \cdot 2 \mathrm{H}_{2} \mathrm{O}$ - and $\mathrm{K}_{2} \mathrm{C}_{2} \mathrm{O}_{4} \cdot \mathrm{H}_{2} \mathrm{O}$-powders. The values of $\alpha$ obtained from the least squares analysis are equal to values found earlier from single crystal NMR-studies. The values of $\beta^{2}$ are in agreement with the values calculated from the proton positions as determined from neutron diffraction investigations.
\end{abstract}

Ry means of proton magnetic resonance spectroscopy it is possible to locate the hydrogen atoms in solids. ${ }^{1}$ The structural information is usually deduced from the second moment of the proton magnetic resonance spectrum which is related to the structure through the Van Vleck formula. ${ }^{2}$ The second moment is a fairly complicated function of the interatomic distances, and, therefore, to get useful information about a structure with more than one degree of freedom it is necessary to have the solid under study as a single crystal. However, due to the limited sensitivity of present day spectrometers the single crystal must have a volume of about 0.1 to $1 \mathrm{~cm}^{3}$, which greatly reduces the use of this technique. ${ }^{3}$

We have therefore been tempted to explore further the line shape analysis given by Pake ${ }^{4}$ in 1948 of the spectra of pair-powders, i.e. powders where all the resonant nuclei are situated in equivalent pairs with the intrapair magnetic dipole-dipole coupling stronger than the coupling to the remaining nuclei. Pake showed that the theoretical line shape of pair-powders could be approximately calculated on the basis of only two parameters, and he showed that the calculated spectra were in qualitative agreement with experimental spectra. 
The theoretical line shape function given by Pake has been used sporadically in the literature, ${ }^{5}$ but no systematic analysis of the possibilities and limitations of the function seems to have been made. ${ }^{6}$ This might be due to computational difficulties in the early days of NMR-spectroscopy, but these difficulties are greatly reduced today using modern computers.

The attracting feature of the Pake function is that it opens up the possibility of determining uniquely the intrapair distance from a powder spectrum. If the Pake function could be used successfully it should therefore be possible to divide, unambiguously, the second moment into an intrapair part and an interpair part.

A powder NMR-spectrum is easily recorded, and as we are going to show, it is also easy to fit the shape function to the experimental spectrum by the method of least squares. The real test of the method to be proposed will be the application in actual cases. The theoretical line shape has been fitted to a series of spectra obtained in our laboratory, and the Pake function will be shown to reproduce the experimental spectra remarkably well. At the moment only the analysis of the spectra of some perhydrates ${ }^{7}$ and $\mathrm{KHCO}_{3}{ }^{8}$ have been completed, but we are working on the analysis of spectra of hydrates, sulfamates, and some compounds containing fairly isolated $\mathrm{CH}_{2}$-groups, and the results of these studies will be published later. Here, we will present the method and discuss different sources of systematic errors of experimental and theoretical origin. The spectra of two hydrates where the hydrogen atom positions have been determined earlier by other methods will be analyzed to give an indication of the reliability and accuracy of the powder-pair method.

\section{EXPERIMENTAL}

The proton magnetic resonance spectra were recorded on a Dual Purpose NMR Spectrometer from Varian Asc. operating at $60 \mathrm{MHz}$, equipped with a low proton background probe (V 4331 LP) and a 12" Varian magnet. The magnetic field was swept with a Field dial unit also from Varian Asc. The scanning speed was from $0.3 \mathrm{G} / \mathrm{min}$ to 2.5 $\mathrm{G} / \mathrm{min}$ with corresponding time constants from $12 \mathrm{sec}$ to $0.8 \mathrm{sec}$. Maximum field amplitude $\left(H_{1}\right)$ was $1 \mathrm{mG}$ or lower depending on the sample.

About five spectra were analyzed of each compound, and the values of $\alpha$ and $\beta^{2}$ to be given are the mean values of the obtained results. The standard deviation of these mean values have been calculated from the set of $\alpha^{\prime}$ s and $\beta^{2}$ 's, and not directly from the sum of the least squares residues. The given standard deviation must therefore be regarded as a measure of the spectral reproduceability.

The second moment calculated from the proton positions was calculated by means of a computer program written for Univac 1107.

\section{THEORETICAL LINE SHAPE}

The theoretical line shape for a powder consisting of magnetic nuclei grouped in structurally equivalent pairs has been shown by Pake ${ }^{4}$ to be given by:

$$
F(h)=\int_{-\infty}^{\infty}\left(h-h^{\prime}\right) g\left(h^{\prime}\right) \cdot S\left(h-h^{\prime}\right) \mathrm{d} h^{\prime}
$$

Acta Chem. Scand. 22 (1968) No. 2 
where $h$ is the magnetic field measured from the midpoint of the spectrum.

$$
\begin{aligned}
& g(h)=C_{1}(1-h / \alpha)^{-1 / 2}+C_{2}(1+h / \alpha)^{-1 / 2} \\
& C_{1}=1 \text { when }-2 \alpha \leq h \leq \alpha \text { otherwise } C_{1}=0 \\
& C_{2}=1 \text { when }-\alpha \leq h \leq 2 \alpha \text { otherwise } C_{2}=0 \\
& \alpha=\left\langle\frac{3 \mu}{2 R^{3}}\right\rangle
\end{aligned}
$$

Here $\mu$ is the magnetic dipol moment of a proton, and $R$ is the intrapair protonproton distance properly averaged over the motion of the pair in the lattice. Eqn. (1) is the derivative of the function given by Pake. The derivative is used here, because this function is directly comparable to the experimental spectra obtained using standard modulation technique.

The broadening of the distribution function, $g(h)$, is taken into account by the function $S(h)$ which, somewhat arbitrarily, is chosen by $\mathrm{Pake}^{4}$ as

$$
S(h)=\exp \left(-h^{2} /\left(2 \beta^{2}\right)\right)
$$

The second moment of eqn. (3) has been shown by Look, Lowe, and Northby ${ }^{9}$ to be

$$
M_{2}=\frac{4}{5} \alpha^{2}+\beta^{2}
$$

The second moment of a powder is given by the Van Vleck formula. ${ }^{2}$

where

$$
M_{2}=M_{2 \mathrm{e}}+M_{2 \mathrm{a}}
$$

$$
\begin{gathered}
M_{2 \mathrm{a}}=\frac{4}{5} \alpha^{2} \\
M_{2 \mathrm{e}}=\frac{2}{3}\left(\frac{3 \mu}{2}\right)^{2} \sum_{\mathrm{i}} r_{\mathrm{i}}^{-6}+\frac{4}{15} \sum_{\mathrm{k}} \mu_{\mathrm{k}}^{2} \frac{I_{\mathrm{k}}+1}{I_{\mathrm{k}}} r_{\mathrm{k}}^{-6}
\end{gathered}
$$

Here $M_{2 \mathrm{a}}$ is the intrapair part of the second moment and $M_{2 e}$ the interpair part, $r_{\mathrm{i}}$ is the distance between any two protons not in the same pair, $r_{k}$ is the distance between one proton and any other nucleus in the structure carrying a magnetic dipole moment, $\mu_{\mathrm{k}}$, and spin, $I_{\mathrm{k}}$. The first term in the equation for $M_{2 e}$ has been multiplied in this pair case by $\frac{5}{6}$ as first shown by Reif and Purcell, ${ }^{10}$ and discussed by Pedersen.11

Comparing eqns. (4) and (5) it follows that

$$
\beta^{2}=M_{2 \mathrm{e}}
$$

Eqn. (5) is valid for a static configuration of nuclei. If there is restricted motion of some kind, as found in many hydrates, eqn. (5) must be altered. Eqn. (6), however, is still valid within the approximation introduced with eqn. (3). In appendix $I$ is given an expression for $M_{2 \mathrm{e}}$ valid for an ensemble of flipping pairs.

From eqns. (1), (2), and (3) it can be seen that the line shape, $F(h)$, depends on only two parameters, $\alpha$ and $\beta$. From the functional form of $F(h)$ it follows that $\alpha$ is a scale factor and the ratio $\beta / \alpha$ determines the shape of the spectrum. We have calculated $F(h)$ for a series of $\beta / \alpha$-values, and typical spectra are shown in Fig. 1. From this figure it can be seen that with $\beta / \alpha<0.30$ the 
spectrum has a very characteristic shape consisting of a central doublet with two smaller peaks on the outer tails of the doublet. At $\beta / \alpha=0.40$ the two outer peaks have disappeared in the tails, and for increasing values of $\beta / \alpha$ also the doublet structure disappears. When $\beta / \alpha>0.70$ the spectrum is without fine structure and equal to the spectrum of a uniform distribution of atomic nuclei.

\section{LEAST SQUARES ANALYSIS}

To fit a theoretical line shape to an experimental spectrum we have used the following procedure. We draw a base line through the spectrum, and choose an origin on the base line outside the spectrum. From this origin we measure the value of the spectrum, $y_{\mathrm{i}}$, at certain points, $h_{\mathrm{i}} \cdot y_{\mathrm{i}}$ is measured in arbitrary units, $h_{\mathrm{i}}$ is measured in gauss. The chosen points, usually about 25 , are distributed evenly over the spectrum. We then minimize the sum

$$
\sum\left[N \cdot F\left(h_{\mathrm{i}}-h_{0}, \alpha, \beta\right)-\left(y_{\mathrm{i}}-y_{0}\right)\right]^{2}
$$

Here $N$ is a normalization factor, $h_{0}$ the distance from the origin to the midpoint of the spectrum, and $y_{0}$ a correction in the base line. (The exact position of the base line and the midpoint of the spectrum is not known, therefore also the parameters determining these quantities are introduced in eqn. (7)).

Eqn. (7) is then minimized using an iteration procedure formulated in a more general least squares program written by Kolboe for Univac $1107 .{ }^{12}$ In the beginning of each cycle the values of $N$ and $y_{0}$ are calculated by means of standard linear regression formulas using the input values of $h_{0}, \alpha$, and $\beta$. The values of $h_{0}, \alpha$, and $\beta$ are then varied independently to minimize eqn. (7). Usually about five cycles are necessary before eqn. (7) is minimized. The starting input values of the parameters are guessed from comparing the experimental spectrum with the spectra in Fig. 1 .

\section{DISCUSSION}

Effect of finite modulation amplitude. The theoretical line shape function (eqn. (1)) is the derivative of the absorption curve. The present day wide line spectrometers are using a modulation technique giving a signal which in the limit of zero modulation amplitude is equal to this derivative. For finite modulation amplitudes the experimental spectrum will deviate from the true derivative, and, hence, deviate from eqn. (1). We have not made any attempts to estimate the effects of using a finite modulation amplitude theoretically, but we have tried to evaluate the effects using an experimental approach. We have recorded the spectrum of powdered gypsum using different modulation amplitudes, $H_{\mathrm{M}}$, and fitted theoretical line shapes to the thus derived experimental spectra. One spectrum obtained at each modulation amplitude is given in Fig. 2. The values we have obtained for $\alpha, \beta^{2}$, and $\beta / \alpha$ are given in Table 1.

From the spectra in Fig. 2 it can be seen that the range of modulation amplitudes covered is large, introducing a marked distortion of the spectra

Acta Chem. Scand. 22 (1968) No. 2 


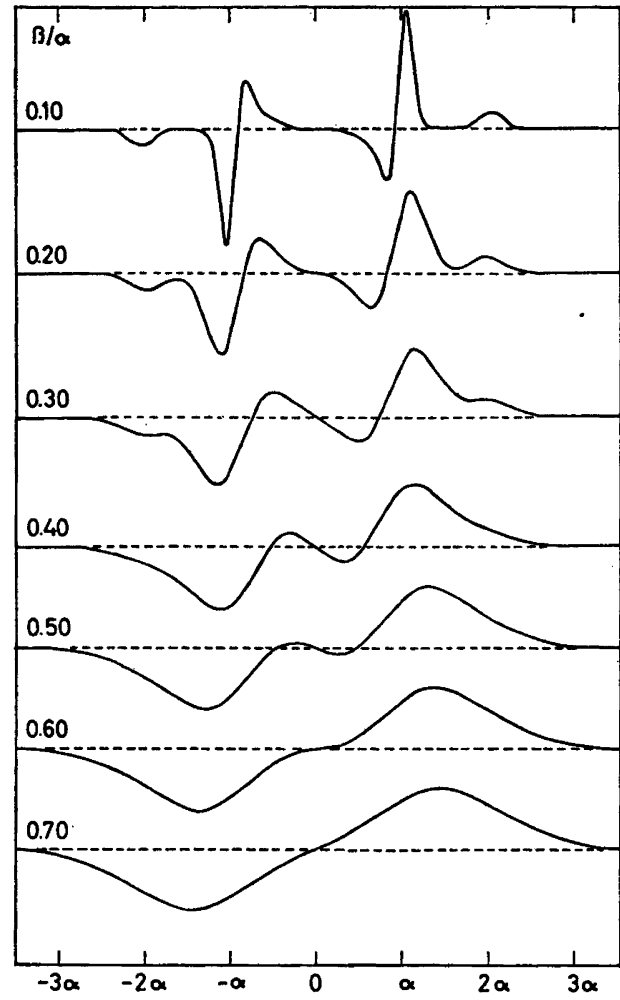

Fig. 1. Theoretical pair-powder NMRspectra.

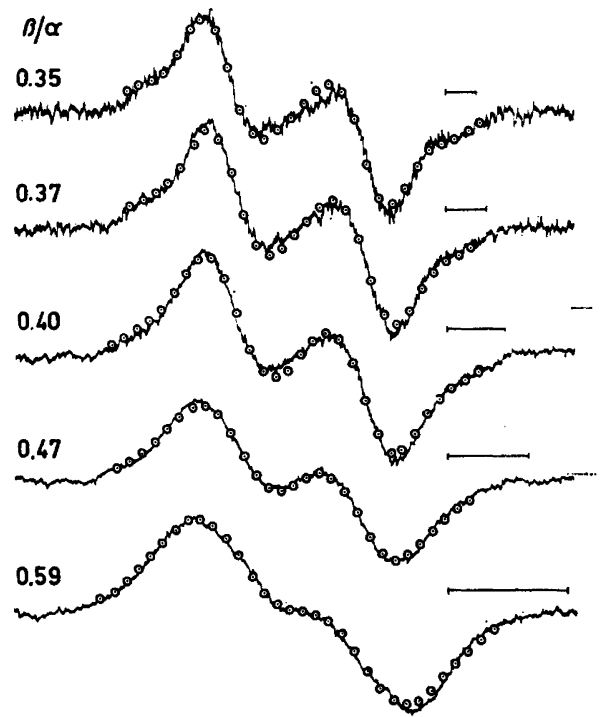

Fig. 2. The experimental pair-powder spectrum of gypsum recorded at different modulation amplitudes. The modulation amplitude (peak to peak) is given in the right corner of each spectrum. The points represent the least-squares fitted theoretical spectrum.

at the higher modulation amplitudes. The theoretical line shape, however, reproduces the experimental line shapes remarkably well also at the higher modulation amplitudes. (In Fig. 2 is given the calculated spectrum as a discrete number of points to show the density of points commonly used in the least square analysis).

The data in Table 1 tell us that the value of $\alpha$ is independent of $H_{\mathrm{M}}$ up to the modulation amplitude $3.93 \mathrm{G}$ (peak to peak), and for higher modulations $\alpha$ is slightly reduced in magnitude. $\beta / \alpha$ increases almost linearly with $H_{\mathrm{M}}$, and $\beta^{2}$ goes up approximately as $H_{\mathrm{M}}{ }^{2}$. Due to the lack of a theoretical basis it is difficult to extrapolate $\alpha, \beta / \alpha$, and $\beta^{2}$ to zero modulation amplitude. But using the fact that $\beta^{2}$ is equal to the interpair part of the second moment, it is easy to explain the increase in $\beta^{2}$ with $H_{\mathrm{M}}$ as the well known modulation 
Table 1. The line shape parameters as function of modulation amplitude in gypsum. (Standard deviation in parenthesis).

\begin{tabular}{|c|c|c|c|c|c|}
\hline $\begin{array}{r}2 H_{M} \\
(G)\end{array}$ & $\stackrel{\alpha}{a}$ & $\beta / \alpha$ & $\begin{array}{c}\beta^{2} \\
\left(\mathrm{G}^{2}\right)\end{array}$ & $\begin{array}{c}\left(\frac{1}{2} H_{\mathrm{M}}\right)^{2} \\
\mathrm{G}^{2}\end{array}$ & $\beta_{0}{ }^{2}$ \\
\hline 1.38 & $\begin{array}{c}5.058 \\
(0.035)\end{array}$ & $\begin{array}{c}0.348 \\
(0.006)\end{array}$ & $\begin{array}{c}3.16 \\
(0.05)\end{array}$ & 0.10 & 3.06 \\
\hline 1.86 & $\begin{array}{c}5.053 \\
(0.021)\end{array}$ & $\begin{array}{c}0.354 \\
(0.003)\end{array}$ & $\begin{array}{c}3.20 \\
(0.04)\end{array}$ & 0.22 & 2.98 \\
\hline 2.61 & $\begin{array}{c}5.088 \\
(0.013)\end{array}$ & $\begin{array}{c}0.370 \\
(0.003)\end{array}$ & $\begin{array}{c}3.54 \\
(0.05)\end{array}$ & 0.43 & 3.11 \\
\hline 3.93 & $\begin{array}{c}4.994 \\
(0.012)\end{array}$ & $\begin{array}{c}0.403 \\
(0.003)\end{array}$ & $\begin{array}{c}4.04 \\
(0.05)\end{array}$ & 0.97 & 3.07 \\
\hline 5.46 & $\begin{array}{c}4.945 \\
(0.017)\end{array}$ & $\begin{array}{c}0.470 \\
(0.002)\end{array}$ & $\begin{array}{c}5.41 \\
(0.03)\end{array}$ & 1.83 & 3.58 \\
\hline 7.84 & $\begin{array}{c}4.878 \\
(0.012)\end{array}$ & $\begin{array}{c}0.592 \\
(0.003)\end{array}$ & $\begin{array}{c}8.33 \\
(0.05)\end{array}$ & 3.84 & 4.49 \\
\hline
\end{tabular}

correction $^{13}$ in the second moment: $\left(\frac{1}{2} H_{\mathrm{M}}\right)^{2}$. This modulation correction has been given in Table 1, and it can be seen that, except for the two highest modulation amplitudes, will the modulation correction properly account for the increase in $\beta^{2}$ with $H_{\mathrm{M}}$.

From this experiment we have drawn the following conclusion which also is supported from our general experience with the use of the powder-pair method: If we use a modulation amplitude which does not drastically alter the shape of the spectrum, the obtained value of $\alpha$ is independent of the modulation amplitude, and the obtained value of $\beta^{2}$ is corrected for modulation broadening by subtracting the modulation correction:

$$
\beta_{0}^{2}=\beta^{2}-\left(\frac{1}{2} H_{\mathrm{M}}\right)^{2}
$$

where $\beta_{0}{ }^{2}$ is the broadening parameter due to the interpair interaction.

\section{EXPERIMENTAL ILLUSTRATIONS}

To give an indication of the inherent possibilities of the powder-pair method as outlined above, we will now analyze the spectra of two hydrates: $\mathrm{K}_{2} \mathrm{C}_{2} \mathrm{O}_{4} \cdot \mathrm{H}_{2} \mathrm{O}$ and $\mathrm{CaSO}_{4} \cdot 2 \mathrm{H}_{2} \mathrm{O}$ (gypsum). We have looked in the literature for

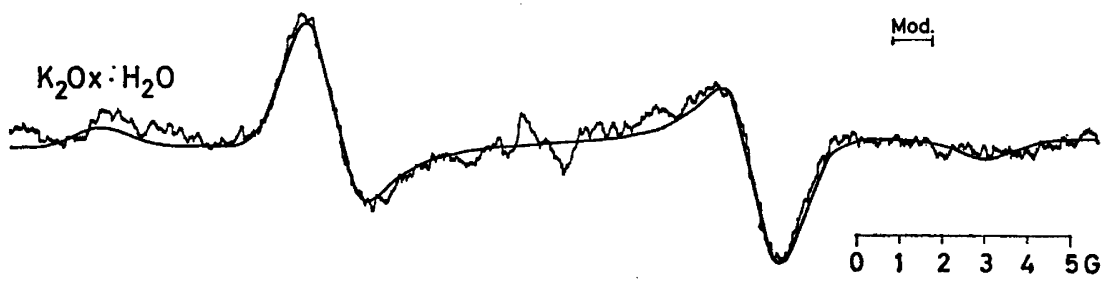

Fig. 3. The experimental pair-powder spectrum of potassium oxalate monohydrate with the least-square fitted spectrum superimposed (smooth curve).

Acta Chem. Scand. 22 (1968) No. 2 
other test samples, where the proton configuration had been determined unambiguously by means of other methods, but these two hydrates are the only ones we have found.

The powder spectrum of gypsum is given in Fig. 2, and the spectrum of $\mathrm{K}_{2} \mathrm{C}_{2} \mathrm{O}_{4} \cdot \mathrm{H}_{2} \mathrm{O}$ in Fig. 3 together with the least-squares fitted theoretical spectra. The values of $\alpha$ and $\beta^{2}$ as obtained from the analysis are given in Table 2 together with earlier determinations of $\alpha$ from single crystal NMR investigations and calculated values of the interpair contribution to the second moment for a flipping and a stationary collection of $\mathrm{H}_{2} \mathrm{O}$-molecules using the proton coordinates found in the neutron diffraction studies.

Table 2. Powder-pair $\alpha$ and $\beta^{2}$ values compared to literature values.

\begin{tabular}{|c|c|c|c|c|c|c|}
\hline \multirow{3}{*}{$\frac{\text { Compound }}{\mathrm{K}_{2} \mathrm{C}_{2} \mathrm{O}_{4} \cdot \mathrm{H}_{2} \mathrm{O}}$} & \multirow{3}{*}{$\begin{array}{c}\text { This work } \\
\begin{array}{c}5.16 \\
(0.01)\end{array}\end{array}$} & \multirow{2}{*}{\multicolumn{2}{|c|}{$\begin{array}{l}\stackrel{\alpha}{(G)} \\
\text { Single crystals }\end{array}$}} & \multirow{3}{*}{$\begin{array}{c}\text { This work } \\
\begin{array}{c}0.30 \\
(0.01)\end{array}\end{array}$} & \multicolumn{2}{|c|}{$\begin{array}{c}\boldsymbol{\beta}^{\mathbf{2}} \\
\left(\mathrm{G}^{2}\right) \\
\text { Calculated }\end{array}$} \\
\hline & & & & & static & flip \\
\hline & & $5.14^{a}$ & $\begin{array}{c}5.20^{b} \\
(0.02)\end{array}$ & & $0.34^{d}$ & 0.23 \\
\hline $\mathrm{CaSO}_{4} \cdot 2 \mathrm{H}_{2} \mathrm{O}$ & $\begin{array}{c}5.06 \\
(0.03) \\
\end{array}$ & $5.0^{c}$ & & $\begin{array}{c}3.04 \\
(0.05) \\
\end{array}$ & $4.98^{e}$ & 3.05 \\
\hline
\end{tabular}

a Chidambaram, R. as quoted by El Saffar, Z.M. J. Chem. Phys. 45 (1966) 4643.

$b$ Pedersen, B. Acta Cryst. 20 (1966) 412.

c Pedersen, B. and Holcomb, D. F. J. Chem. Phys. 38 (1963) 610.

d Chidambaram, R., Sequeira, A., and Sikka, S. K. J. Chem. Phys. 41 (1963) 3616.

- Atoji, M. and Rundle, R. E. J. Chem. Phys. 29 (1958) 1306.

Inspection of the data in Table 2 shows that the values of $\alpha$ obtained from the powder spectrum are in remarkable good agreement with the values of $\alpha$ found in earlier single crystal studies. This agreement is somewhat surprising as it is known that the interpair coupling produces an asymmetric broadening of the line components in the single crystal spectra of these compounds. ${ }^{14}$ We have assumed symmetric broadening, but, apparently, the averaging over the powder seems to eliminate any systematic error from this source.

From $T_{1}$-measurements in $\mathrm{CaSO}_{4} \cdot 2 \mathrm{H}_{2} \mathrm{O}$ it is known that the water molecules are flipping fast at room temperature..$^{15}$ The measured value of $\beta^{2}$ is also found to be in excellent agreement with such a model and significantly smaller than the static value (see Table 2). In $\mathrm{K}_{2} \mathrm{C}_{2} \mathrm{O}_{4} \cdot \mathrm{H}_{2} \mathrm{O}$ it is not known with certainty whether the water molecules are flipping fast enough at room temperature or not to have an effect on the interpair second moment. The difference between the static and the flipping $\beta^{2}$ is fairly small, and it is not possible to judge from the data given here whether the dynamic or the static model applies. McGrath and Paine ${ }^{16}$ have calculated the potential hindering the flipping motion to $14 \mathrm{kcal} / \mathrm{mole}$. If this is correct a simple estimate shows that the water molecules are not flipping fast enough at room temperature to affect the line components. 
The calculated values of $\beta^{2}$ are not sensitive to small changes in the proton positions. ${ }^{7}$ The noted agreement between the observed and calculated values of $\beta^{2}$ is therefore more a support for the interpretation of $\beta^{2}$ as $M_{2 \mathrm{e}}$ than a support for the given proton positions.

\section{CONCLUSIONS}

We have in this paper described a least squares method to fit a theoretical spectrum to an experimental proton magnetic resonance spectrum of a pairpowder. We have used the expression for the theoretical spectrum as given by Pake, ${ }^{4}$ which rests on many simplifying assumptions in treating the interpair coupling. Pake assumes that the coupling produces a symmetric broadening of the interpair spectrum, that the broadening is gaussian, and that the interpair coupling is isotropic. None of these assumptions are strictly valid in an actual case. However, we have found that the theoretical spectrum gives a very accurate description of the shape of the experimental spectra of pairpowders. Furthermore, in the two experimental illustrations given here, the physical significance of the parameters in the theoretical spectrum is clearly demonstrated. Apparently, therefore, the simplifying description of the interpair coupling does not lead to large systematic errors. However, only detailed studies of a variety of pair-powders can give us a better understanding of the limitations of the pair-powder method. At the moment this method seems to open up the possibility of a systematic study of the variation in the intrapair coupling with environment in a series of compounds without any experimental limitations on the choice of samples.

Note added in proof. A recent calculation has shown that the reduction factor 5/6 included in the equations given above for $\mathbf{M}_{2 \mathrm{e}}$ should not be applied. (B. Pedersen, Chem. Phys. Letters 1 (1967) 373). The equations for $M_{2}$ given above and the calculated numerical values for $\beta_{0}{ }^{2}$ given in Table 2 should therefore be multiplied by 6/5. The calculated values of $\beta_{0}{ }^{2}$ are then somewhat larger than the observed values.

\section{APPENDIX \\ THE INTERPAIR PART OF THE SECOND MOMENT}

We will in this appendix develop the interpair part of the second moment valid for a powder in which the pairs are executing fast $180^{\circ}$ flip motion.

The interpair part of the second moment of a single crystal consisting of flipping pairs has been calculated earlier. ${ }^{11}$

where

$$
\begin{aligned}
& M_{2 \mathrm{e}}=\frac{5}{6}\left(\frac{3 \mu}{2}\right)^{2} \cdot \frac{1}{8} \sum_{\mathrm{j}}\left(b_{11, \mathrm{j} 1}+b_{11, \mathrm{j} 2}+b_{12, \mathrm{j} 1}+b_{12, \mathrm{j} 2}\right)^{2} \\
& +\frac{1}{12} \sum_{\mathrm{i}}\left(\gamma_{\mathrm{i}} h\right)^{2} I_{\mathrm{i}}\left(I_{\mathrm{i}}+1\right)\left(b_{11, \mathrm{i}}+b_{12, \mathrm{i}}\right)^{2}
\end{aligned}
$$

and

$$
b_{1 \mathrm{k}, \mathrm{j} 1}=r_{1 \mathrm{k}, \mathrm{j} 1}{ }^{-3}\left(3 \cos ^{2} \theta_{1 \mathrm{k}, \mathrm{j} 1}-1\right)
$$

$$
b_{1 \mathrm{k}, \mathrm{i}}=r_{1 \mathrm{k}, \mathrm{i} 1}{ }^{-3}\left(3 \cos ^{2} \theta_{1 \mathrm{k}, \mathrm{i}}-1\right)
$$

Acta Chem. Scand. 22 (1968) No. 2 
Here we put pair 1 , with protons 11 and 12 , at the origin, and calculate the contributions from the pair $\mathrm{j}$, with protons $\mathrm{j} 1$ and $\mathrm{j} 2$. The interatomic distance between say 11 and $\mathrm{j} 2$ is $r_{11, \mathrm{j} 2}$, which is making an angle $\theta_{11, \mathrm{j} 2}$ with the magnetic field. The term $b_{1 \mathrm{k}, \mathrm{j}}$ is the similar contribution from the nucleus $\mathrm{i}$ with gyromagnetic ratio $\gamma_{\mathrm{i}}$ and spin $I_{\mathrm{i}}$.

To get $M_{2 e}$ for a powder we must average the equation above over a sphere. (We assume that we can keep the term 5/6 for all orientations, and ignore the complications introduced when the pair approximation breaks down ${ }^{\mathrm{II}}$ ). The averaged expression gets very long and complicated, and will not be given here, but a general term in the expression will be $\left\langle b_{1 \mathrm{k}, \mathrm{j} 1} \cdot b_{11, \mathrm{i} 1}\right\rangle$. Carrying out the average it is easy to show that:

$$
\left\langle b_{1 \mathrm{k}, \mathrm{j} 1} \cdot b_{11, \mathrm{i} 1}\right\rangle=\frac{1}{5} r_{1 \mathrm{k}, \mathrm{j} 1}{ }^{-3} \cdot r_{11, \mathrm{i} 1}{ }^{-3}\left(3 \cos ^{2} \alpha_{1 \mathrm{k}, \mathrm{j} 1} \dot{11}_{1, \mathrm{i} 1}-1\right)
$$

where $\alpha_{1 \mathrm{k}, \mathrm{j} 1}: 11, \mathrm{i} 1 \mathrm{is}$ the angle between $r_{1 \mathrm{k}, \mathrm{j} 1}$ and $r_{11, \mathrm{i} 1}$.

Acknowledgement. This work has been supported by The Royal Norwegian Council for Scientific and Industrial Research. Our thanks are also due to S. Kolboe, E. Mehlum, and T. Bråthen for indispensable aid in working out the computer program, and to Mrs. M. Jørve for technical assistance.

\section{REFERENCES}

1. Aleksandrov, N. M. and Skripov, F. I. Soviet Phys.-Usp. (English Transl.) 4 (1962) 947.

2. Van Vleck, J. H. Phys. Rev. 74 (1948) 1168.

3. Dereppe, J. M., Touillaux, R. and Van Meerssche, M. J. Chem. Phys. 63 (1966) 1265.

4. Pake, G. E. J. Chem. Phys. 16 (1948) 327.

5. Freeman, R. and Richards, R. E. Trans. Faraday Soc. 52 (1956) 802;

Richards, R. E. and York, R. W. Trans. Faraday Soc. 54 (1958) 321.

6. Forman, R. A. and Kahn, A. H. J. Chem. Phys. 45 (1966) 4586.

7. Pedersen, B. F. and Pedersen, B. Selected Topics in Structure Chemistry, Oslo University Press, Oslo 1967, p. 253.

8. Pedersen, B. To be published in Acta Cryst.

9. Look, D. C., Lowe, I. J. and Northby, J. A. J. Chem. Phys. 44 (1966) 3441.

10. Reif, I. and Purcell, E. M. Phys. Rev. 91 (1953) 631.

11. Pedersen, B. J. Chem. Phys. 39 (1963) 720.

12. Kolboe, S. Ind. Eng. Chem., Fundamentals 6 (1967) 169.

13. Halbach, M. Phys. Rev. 119 (1960) 1230.

14. Holcomb, D. F. and Pedersen, B. J. Chem. Phys. 38 (1963) 54.

15. Holcomb, D. F. and Pedersen, B. J. Chem. Phys. 36 (1962) 3270.

16. McGrath, J. W. and Paine, A. A. J. Chem. Phys. 41 (1964) 3551.

Received June 23, 1967. 